\title{
Multidisciplinary Visual Rehabilitation in Low- and Middle-Income Countries: A Systematic Review
}

\author{
Sarah Wallace ${ }^{1}$, Rotimi Alao ${ }^{2}$, Hannah Kuper ${ }^{3}$, Mary Lou Jackson ${ }^{4}$ \\ ${ }^{1}$ Public Health Training Scheme, London Deanery, UK \\ ${ }^{2}$ MSc Programme, London School of Hygiene and Tropical Medicine, London, UK \\ ${ }^{3}$ London School of Hygiene and Tropical Medicine, London, UK \\ ${ }^{4}$ University of British Columbia Department of Ophthalmology, Vancouver, Canada \\ Correspondence information: hannah.kuper@Ishtm.ac.uk
}

\author{
Declarations of Interest: Mary Lou Jackson is a consultant for Astellas. Hannah Kuper's salary is supported by \\ the PENDA grant from DFID. Sarah Wallace, and Rotimi Alao and have no declarations of interest to declare. \\ Running Head: Visual Rehabilitation in LMICs Systematic Review \\ Article Category: Review Article \\ Word Count: 9,749 (including tables)
}


21 Objective: To systematically identify the evidence for effectiveness of rehabilitation interventions in people who are visually impaired, living in low- and middle-income countries.

23 Methods: Fifteen databases and the grey literature were searched up until February 2020; papers were 24 identified according to eligibility criteria, and assessed for risk of bias. Eligible studies were controlled trials 25 (randomised or non-randomised) of rehabilitation interventions for blind or visually impaired adults or 26 children from low- and middle-income countries. Possible outcomes included visual acuity, activities of daily 27 living, safety, quality of life and psychological status.

28 Results: Fifteen eligible studies were identified from India (7), Turkey (4), Nigeria (2), Croatia (1) and Iran (1). 29 Six studies were randomised, seven were non-randomised trials, and in two the method of allocation was not 30 clear. Participants were adults (6), children (7) and both adults and children (2). Seven studies were small $31(n \leq 65)$ and examining the effect of training programmes. Remaining studies compared the effect of low vision 32 aids (3), economic rehabilitation, goalball, rehabilitation compliance and service delivery methods (2), 33 including one large four-arm randomised trial $(n=436)$. Studies measured a variety of outcomes, and mostly 34 showed a positive effect of interventions for pre- and post-intervention assessment, although between 35 intervention group comparisons were often inconclusive. Overall, only four studies had a low risk of bias.

36 Conclusions: A lack of high-quality evidence for rehabilitation interventions is a barrier to provision of low 37 vision services in low- and middle-income countries. Future research should focus on establishing 38 effectiveness and cost-effectiveness of devices and models of vision rehabilitation appropriate for low39 resource settings.

\section{KEYWORDS}

42 Systematic Review, Low Vision, Blindness, Visual Impairment, Rehabilitation, Low and Middle Income 43 Countries 
There are an estimated 36 million people globally who are blind, and a further 216.6 million with moderate to severe visual impairment, of whom the vast majority live in low- and middle-income countries (LMICS) (1). An estimated $80 \%$ of blindness is avoidable, and ongoing progress in the prevention, early identification and treatment of eye disease is likely to reduce the prevalence of blindness and visual impairment. However, there will remain millions of people with blindness or low vision which is not preventable and/or not treatable. Studies have consistently established that vision impairment severely impacts quality of life (QoL) among adult populations (2), and there is evidence that visual impairment is linked to anxiety and depression (2). Furthermore, living with vision loss can cause difficulties in many areas of life and across the whole life course; this may include delayed early development (3) and lower educational attainment in childhood (4), adverse impact on employment (5) and reduced participation in leisure activities and activities of daily living, such as reading, outdoor mobility and shopping.(6) There is also evidence elderly that elderly living with visual impairment have an increased risk of injury (7), falls $(8,9)$, depression (10), dependence and mortality (11). It is therefore vital that people with irreversible vision loss are offered effective rehabilitation interventions which reduce the adverse effects of this impairment on their lives.

The World Health Organization (WHO) describes rehabilitation as "a set of interventions designed to optimize functioning and reduce disability in individuals with health conditions in interaction with their environment.... Rehabilitation is characterised by interventions that address impairments, activity limitations and participation restrictions, as well as personal and environmental factors (including assistive technology) that have an impact on functioning" (2). Rehabilitation must, therefore, provide a wide range of interventions to address such a range of impacts, and often involve a range of service providers. Multidisciplinary vision rehabilitation interventions include, but are not limited to, devices, training, environmental modifications, psychosocial supports, vocational services, and community services. The importance of visual rehabilitation is increasingly recognised; one of the aims of the universal eye health global action plan 2014-2019 is "to secure access to rehabilitation services for the visually impaired" (12). Rehabilitation of people living with low vision and blindness will also help people fulfil their rights as set out in the United Nations Convention on the Rights of Persons with Disabilities (13), and countries to achieve the Sustainable Development Goals (14). Effective and accessible rehabilitation services for children and adults with low vision will enable access to education (goal four), participation in the labour market (goal eight), reduce inequality thorough increased inclusion in society (goal 10) and improve safety when navigating transport systems (goal 11) (14). The impact of rehabilitation interventions on individuals who are blind or have low vision can therefore be assessed through a broad variety of methods; these include visual acuity testing, activity based measures (such as reading or writing ability, mobility, ability to carry out activities of daily living etc), safety or adverse incident measures (such as falls or accidents), psychological or quality of life measures.

Evidence of effectiveness of interventions of visual rehabilitation interventions is limited. Of eight Cochrane reviews of low vision rehabilitation only three identified eligible studies, and were all in adults (15-17). One assessed reading aids (15) and one investigated orientation and mobility training (16). Both these reviews included only trials from high-income countries, and concluded there was insufficient evidence in this area, although the former paper did find that there is "some evidence that stand-mounted electronic devices may improve reading speeds compared with optical devices" (15). The third review examined the effectiveness of low vision rehabilitation on quality of life measures. The findings showed some evidence of a small benefit of psychological therapies and methods for enhancing vision (e.g. use of assistive technologies or training of residual vision functions) on vision-related quality of life (low and moderate certainty respectively), but no evidence of benefit or very low certainty evidence for the more general health related quality of life measures, or for the impact of multidisciplinary rehabilitation programmes (17). An extensive 2012 systematic review of low vision services (18) critiqued 58 studies (of which the vast majority were in high-income countries), noting 
90 an overall paucity of high quality research; they concluded that rehabilitation services result in improved 91 clinical and functional ability outcomes, but evidence is less clear for effect on mood and quality of life. There 92 was a particular lack of evidence for children and for cost-effectiveness.

93 Data are particularly lacking from Low- and Middle-Income Countries (LMICS). Of the three reviews described 94 above, two included only studies from high-income settings and the third included 3 studies from middle95 income countries and none in lower-income countries. We have identified no previous systematic reviews of 96 visual rehabilitation interventions specifically in LMIC. This lack of data on effectiveness in LMICs is a grave 97 concern, as visual rehabilitation services in LMICs are particularly scarce, and suffer from a severe lack of skilled 98 workers and funding; The global survey that Chiang (2011) undertook found that there is no service in around 99 half of African and Western Pacific region countries (19).

100 This review aimed to systematically identify the evidence for rehabilitation interventions in people who are 101 visually impaired (including blindness and low vision) living in LMICs. 


\section{METHOD}

The review has been reported according to PRISMA guidelines (20). A protocol was not published, but is specified below.

\section{Literature search}

Three search concepts were identified ('rehabilitation', 'visual impairment' and 'low and middle-income countries') and related terms were identified (See Appendix 1 for the Medline search strategy). 15 databases were searched in October 2017: Medline, CINAHL, Embase, Global Health, AIM (African Index Medicus), IMEMR, IMSEAR, LILACS, WHOLIS, WPRIM, Web of Knowledge, Campbell Collaboration library, Cochrane database of systematic reviews, CENTRAL (Cochrane Register of Controlled Trials), and HTA Database (The Cochrane Library). Two authors screened titles, abstracts and full texts for inclusion (one author only screened full texts not in English), and discrepancy at the full-text stage was resolved through discussion.

The database search was updated in February 2020.

Grey literature was searched by one author using Google ${ }^{\mathrm{TM}}$ (first 500 results) and we attempted to identify unpublished work through contacting subject experts in the academic field and in non-governmental organisations working in the field. The references of identified papers were searched, as were the references of systematic reviews in the field of visual rehabilitation. Authors were contacted where necessary to locate papers.

\section{Inclusion/Exclusion criteria}

Table 1 outlines the PICO criteria.

Eligible studies were set in LMICs (according to the World Bank List 2017 (21)) and had participants of any age who have moderate or severe visual impairment or are blind. Interventions were included 'that address impairments, activity limitations and participation restrictions, as well as personal and environmental factors (including assistive technology) that have an impact on functioning' (22). Interventions able to fully correct visual impairment were excluded; these included provision of standard spectacles, contact lenses, medication or surgery. Randomised or non-randomised controlled trials were eligible, and they needed to report separately on outcomes in those who are visually impaired. Studies were eligible if they reported one or more of the following outcomes: visual acuity, functional ability to carry out activities of daily living, safety, quality of life, or psychological outcomes. We did not specify a required length of follow-up. Studies had to be available in full text, peer reviewed and published in the last 20 years (1997-current). There were no language restrictions (our protocol planned to include only papers which had title and/or abstracts available in English, but we were able to obtain translations of all titles/abstracts as required).

Exclusion criteria included qualitative studies, studies lacking control groups, studies with a very small sample size (i.e. fewer than 10 participants), and where we were unable to obtain a full-text/full-text not available. Very small studies are unlikely to be able to document and statistically significant impact, and so publication of studies with positive results are likely to be due to chance and publication bias. We therefore restricted our sample size to 10 participants as above, in line with other reviews (18).

\section{Data Extraction and Bias Assessment}

One author undertook data extraction into a standard table and the bias assessment (SW), which were then checked by a second author (HK/RA). We used the Cochrane risk of bias tool (23); where studies were not randomised they scored a high risk of bias in the 'random sequence generation' and 'allocation concealment' criteria, and studies scored moderate risk of bias for the 'blinding of outcome assessment' criteria if the assessors but not the participants, were masked. We contacted authors by email where information regarding 
144 risk of bias was missing. Reporting of results was undertaken narratively, with the expectation that 145 intervention, settings and study designs were too divergent to undertake a meta-analysis. 


\section{RESULTS}

The database search is outlined in Figure 1; 9,424 items were identified, of which 114 full texts were screened. Fifteen papers met the eligibility criteria: fourteen journal articles, and one PhD thesis (24) (the protocol had been published separately (25)). Of these eleven were identified from the database search (26-34) and four from elsewhere (including the PhD related to a paper identified through the search which was sent by the author $(24)$, and two from systematic reviews $(35,36)$ ). We were unable to obtain a translation of one fulltext (37). Table 2 details the included studies.

\section{Population}

The 15 included studies were all undertaken in middle-income countries (India (7 studies), Turkey (4 studies), Nigeria (2 studies), Croatia (1 study), Iran (1 study)). Of the seven trials in India, five were undertaken in Hyderabad $(24,30,31,33,38)$, and one of these was located in both the UK and India (33). Seven studies included children only $(26-28,30,33,36,39)$, with a total number of participants ranging between 20 and 183 , and five of which recruited children from special schools/schools for the blind (26-28, 36, 39). Six studies included adults only $(31,32,34,35,38,40)$, of which one was a group of 60 blind and visually impaired 20-40 year olds who previously had full vision (35), one among a group of participants with macular degeneration $(\mathrm{N}=100)(31)$, one was among 28 war veterans (32), one among university students (40) and the final two recruited participants from a hospital clinic $(\mathrm{N}=255)$ (38) or community $(\mathrm{N}=159)$ (34). Two studies included both adults and children $(24,29)$. including a large study of 436 patients in a tertiary eye care facility (24).

\section{Design and Intervention}

Six studies had a randomised design $(24,26,27,33,35,40)$, seven had a non-randomised controlled design $(28-32,34,38)$ (including one cross-sectional study of goalball players versus non-players (28)), and in two studies the method of allocation was not clear $(36,39)$. There was a wide range of interventions, although seven of the studies took the form of a training programme $(26,27,32,35,36,39,40)$. The training programmes in children delivered motor skills training (physiotherapist administered vs home training) (39), an individually adapted task-based attention training programme delivered twice per week (control received no intervention) (27), physiotherapist delivered group programme of visual perception training which aims to increase activity performance (comparing paper and pen against computer aided training) (26) and a programme to increase motivation to work comparing goal setting and emotional intelligence interventions, although the content of this training programme was poorly described (36). In adults two training programmes were a Rational Emotive Behaviour Therapy in individuals with depression (one control group received no intervention (40) and in one it was unclear what the control group received (35)), a cognitive behavioural therapy which aims to improve psychological well-being, and a mobility rehabilitation course for veterans (control group received no intervention) (32). The programmes lasted between six weeks (27) and three months $(26,39,40)$ (only four of seven reported this information), and only two studies reported that they followed-up individuals beyond the end of the course $(35,40)$. Three studies looked specifically at the effect of various low vision devices, including tablet computers (33), magnifiers (31) and a variety of low vision aids (29). Five studies delivered comprehensive and often multidisciplinary rehabilitation services $(24,30,31,34$, 38). Two studies compared different service provision or models; these included a four-arm randomised controlled trial comparing centre based, community based and mixed models (24), while the other compared optometry led and non-optometry led services (38). A further before-after controlled study compared the difference between children who were compliant with multidisciplinary low-vision rehabilitation and those that were not (30). Finally Vijayakumar et al. (34) delivered community-based rehabilitation with a subgroup receiving economic rehabilitation, which "focussed on providing skills to run a trade or pursue a profession". 
A wide range of outcomes were assessed using a plethora of measures. Two studies used the L. V. PrasadFunctional Vision Questionnaire II (LVP-FVQ II), $(30,33)$ two used the impact of visual impairment (IVI) questionnaire for adults $(24,38)$, two the IVI for children $(24,33)$, and two used the low vision quality of life (LVQOL) tool $(27,29)$, although a variety of other quality of life and vision-related functioning outcome measured were also used. Quality of life and vision-related functioning were the most common outcomes measured, but other aspects included: visual acuity, motor skills, motor fitness, visual perception, social skills, occupational/activity performance, independent mobility, cognitive function, adaptation to visual loss, levels of motivation to work, irrational beliefs, depression, anxiety and stress, and self-esteem. These represent a mixture of patient-reported and assessor-reported outcomes.

\section{Findings}

Most of the studies' results concentrated on the difference between pre- and post-intervention measurements. The vast majority $(12 / 15)$ of the studies reported evidence of a post-intervention improvement in outcomes for all groups that received an intervention. One study was cross-sectional (28) and one did not report pre-post interventions (32). The last study did not find a significant change in quality of life or functioning with provision of electronic devices (tablets) or with standard care (including optical aids), but the trial was a pilot feasibility trial and was not powered for these secondary outcomes (33).

Of six studies comparing two different interventions, all found a positive overall effect of the interventions. Three of these found no difference between the two groups and one did not compare the outcomes of the two groups (31). The three which did not find any significant difference compared paper and pen vs computer in visual perception training (26), goal setting and emotional intelligence (36), and optometry-led services compared to non-optometry led (30). All four arms in Christy's (24) large randomised trial showed a positive impact, however the author concluded that "a significantly greater improvement was observed in the community-based method that involved family members and the community more than the other methods of service delivery." However, the statistical evidence for this conclusion is not clear. Physiotherapist-delivered training produced significantly better results in five of eight motor skills domains compared to parent taught home-training in a group of children (39).

Nine studies compared intervention to no intervention or usual care; seven of these showed a positive impact of the intervention with a significant difference compared to the control group. These studies examined attention training (27), provision of low vision aids (29), effect of compliance with multidisciplinary rehabilitation therapy (38), two for rational emotive behaviour training $(35,40)$, mobility training course (32) and goalball (28). Two studies showed no difference between intervention and control group: a subgroup analysis of economic rehabilitation versus no economic rehabilitation within a community based rehabilitation programme (34), and provision of optical aids versus standard care in a pilot trial as discussed above (33).

\section{Bias Assessment}

Of the six randomised studies, three were judged to have a low risk of selection bias $(24,26,33)$ (strong methods of random sequence generation and allocation concealment), one had a moderate risk of bias for both random sequence generation and allocation concealment (numbers in envelopes) (27), one had a strong method of randomisation but a moderate risk of bias for allocation concealment (40) and one was not described (35). Other studies were either at high risk of selection bias as they were non-randomised (28-32, 34,38 ) or had no description of the allocation process $(36,39)$. While participant blinding/masking would not always have been practicable, only six studies undertook assessor masking (24, 28-30), six did not describe masking $(26,31,32,34-36,39)$, three confirmed that assessment was undertaken unmasked $(27,33,34)$, and the final study stated that both participants and data assessors were masked but this appears to have been solely referring to the pre-intervention assessment (40). Of the thirteen studies where a bias assessment due 
233 to incomplete outcome data was applicable, five studies reported no attrition $(26,27,34,36,40)$ and two had 234 low rates of attrition (29, 33), while two had insufficient information to make a judgement $(32,39)$. Christy's 235 four-arm randomised trial had a 10\% overall loss to follow-up was scored as having moderate risk of bias 236 related to different loss to follow-up between the different study arms (24\% in the centre-based arm). Two 237 studies which recruited patients from hospital clinics had very high rates of loss to follow-up (54\% or greater) 238 (30, 38). One study was scored as having moderate risk of bias from incomplete data as some outcome 239 measures had a patient missing, but with no explanation (35). Twelve of the studies were judged low risk of 240 selective reporting $(24,27-31,33,35,36,38-40)$, while one was judged to be moderate risk as between group 241 comparisons were not fully reported (26), one did not report the between group comparison except in the 242 abstract (34), and one had insufficient information to judge (32). In terms of other biases, one assessment of 243 devices was funded by the device manufacturer (33), while another reported paying the travel expenses to 244 attend follow-up of the intervention group but not to the control group (40). A formal assessment of 245 publication bias was not possible due to heterogeneity. Only four studies have low risk in at least three of the 246 five bias domains $(24,26,33,40)$. 


\section{DISCUSSION}

248 Only 15 eligible controlled trials of rehabilitation interventions in LMICs were identified, highlighting the other trials identified were small and/or of low methodological quality. The studies generally showed a positive impact of rehabilitation in the lives of people with blindness/low vision, but concerns about the quality of the studies and the sparsity of the data available, means that positive conclusions of impact are premature.

Two randomised studies using Rational Emotive Behaviour Therapy demonstrated an improvement in depression symptoms $(35,40)$. These results are consistent with the findings from van Nispen's 2020 Cochrane review on visual rehabilitation and quality of life, which concluded that there is moderate certainty evidence that psychological therapies have an impact on depression (secondary outcome) (17). While Jalili (35) gave little detail, it is clear that Onuigbo's (40) intervention was resource intensive; it required trained professionals to deliver and lasted for 12 weeks excluding follow-up, which produce concerns about the feasibility of this as a model in LMICs. It is imperative that models which are evidence-based, but also viable in terms of cost, time, professional skills are tested and that services working with individuals with visual impairment are able to assess for depression and refer where services are available. It was common that the training courses were delivered by trained therapists; given the known paucity of rehabilitation professionals in LMICs, services should consider whether effective interventions involving delivery of training courses can be delivered by other cadres (e.g. non-clinicians or volunteers). While depression is known to be common among elderly people with vison loss, the two studies were both in younger adults (students and 20-40 years); models need to be evaluated among the elderly, who compose the majority of those who are visually impaired worldwide (12), and who may respond differently to interventions for depression.

The limited evidence base is unsurprising giving the lack of evidence on effectiveness of visual rehabilitation interventions in high-income settings. The dearth of studies in LMICs is also consistent with the scarcity of low vision services in these settings (19). A 2008 review of low vision service provision reported that there was no services or very low/poor coverage in most of the African region and $52 \%$ of countries in the Western Pacific had no services (19). The multiple outcome measures found in our review is consistent with findings in other reviews; Binns et al. (18) identified 46 different outcome measures in the 58 studies in their review of low vision services. Standardising outcome measures in visual rehabilitation research would make comparing and synthesising results easier, which is especially important where many studies have small numbers of participants. Many of the studies assess general outcomes (e.g. quality of life) or composite function outcomes (e.g. Impact of Visual Impairment questionnaire), often in a heterogeneous group, however, reporting on outcomes on specific functional limitations (e.g. reading, educational attainment, employment, ability to shop independently), perhaps as secondary outcomes, might help results to be of more practical use and more easily tailored to individuals' needs.

The studies do not provide clarity on the optimal means for providing rehabilitation to people with visual impairment. When considering different service models, one relevant UK based trial found no evidence of a difference in outcome between enhanced services including supplementary home based low vision rehabilitation and conventional hospital based rehabilitation (with or without with home visits that did not include rehabilitation) (41). While this differs from Christy's (24) tentative conclusion of a greater effect of community-based methods, contextual differences such as transport links and proportions of rural versus urban populations, may explain the differences observed. However, this uncertainty means that caution is needed before advocating for potentially more expensive community-based services over centre-based services. It is vitally important that national eye health programmes consider how rural populations can effectively and sustainably be served and that these are evaluated; there is evidence that a country having a higher proportion of rural dwellers is predictive of having lower coverage of low vision services (42), and patients living in rural areas are less likely to access visual rehabilitation services (19). Most of the studies 
were centre-based and therefore required travel to a central location, which could pose a problem for countries with a high proportion of rural dwellers and/or poor transport links; this is consistent with a systematic review of access to rehabilitation for people with disabilities, where 'logistical factors (distance to service, lack or cost of transport)' were some of the most commonly reported barriers (43).

Four of the studies examined effectiveness of low vision devices $(24,30,31,33,44)$, of which only one was a randomised trial. Low vision devices can be expensive and evidence of effectiveness is important to determine which devices are appropriate for which patients. Sources of lower-cost devices can be identified such as the Vision 2020 Low Vision Resource Centre, which makes low-cost, typically simple optical devices sourced locally, available to send to services worldwide (28). The current trend of increased use of off-the-shelf technologies, such as mobile phones, tablets and digital books as devices to magnify or access text or information, is a move away from reliance on specialist low vision devices, and therefore a shift from an expensive niche market pricing to the possibility of harnessing the affordability of the open market.

There were specific concerns that need to be taken into account when evaluating the findings from the review. We chose to take a wide view of what interventions were considered rehabilitation; visual impairment is known to impact many areas of activities and participation, and therefore we included interventions such as economic rehabilitation. However, there may be differences of opinion regarding interventions which constitute visual rehabilitation. This was a comprehensive systematic review which searched a large number of databases and other sources of publications. However, four of 15 papers were identified outside the database search, potentially due to the challenge of generating search terms that covered the broad range of possible interventions included under "rehabilitation". The risk of reporting bias was high, due to the combination of subjective outcomes, difficulty of masking participants and lack of assessor masking in most studies. Furthermore, follow-up of more than one month beyond the end of the intervention was rarely reported, and therefore it is unclear if the positive outcomes were sustained. The lack of consistency in outcome measures and tools used in the studies makes comparison between interventions and building an evidence base difficult. In terms of generalisability, while studies were identified in a range of countries none of these were in the low-income group, and 11 of the 15 were from India and Turkey. The selection of studies identified is not representative of the population with visual impairment in LMICs; the majority of individuals who live with visual impairment worldwide are older adults, nearly half of the studies are among children only, while five of 15 featured older adults (one unknown (32)), with five of the studies in children from schools for the blind which includes all four of the studies from Turkey. While we could not assess publication bias formally, it seems likely that the risk is high; almost all included studies described positive outcomes. We tried to reduce this as much as possible through searching grey literature and contacting subject experts.

There were also important strengths to the review. We searched 15 databases and used gold standard approaches for screening eligible papers and extracting data, led by a group knowledgeable about systematic reviews and visual rehabilitation.

The review clearly highlights the need for higher quality evidence, with studies using more consistent outcome assessments. Research needs to address not only the effectiveness of devices, but also models of care, dose and timing of interventions, and cost-effectiveness, and must take into account the specific needs of LMIC settings. Research into visual impairment is challenging due to expense, practical difficulties such as masking, heterogeneity of populations with visual impairment, but also due to ethical difficulties in denying a control group a rehabilitation intervention. However as increasing research is carried out in high-income settings in the future, techniques which are found to be effective may be transferrable to LMIC health systems. 
336 Low quality evidence for rehabilitation interventions of those who are visually impaired and blind makes 337 advocacy for visual rehabilitation difficult, and is likely to contribute to the scarcity of service provision in 338 LMICs. While some studies, such as use of Rational Emotive Behavioural Therapy, show favourable results 339 their scalability in many LMIC settings and use in different groups of patients must be considered carefully. 340 Improved evidence for effective and affordable low vision rehabilitation interventions is required to both 341 convince policy makers and patients of the importance of access to rehabilitation services for the visually 342 impaired and to plan and implement services. 
1. Bourne RRA, Flaxman SR, Braithwaite T, Cicinelli MV, Das A, Jonas JB, et al. Magnitude, temporal trends, and projections of the global prevalence of blindness and distance and near vision impairment: a systematic review and meta-analysis. Lancet Glob Health. 2017;5(9):e888-e97. Epub 2017/08/07. doi: 10.1016/s2214-109x(17)30293-0. PubMed PMID: 28779882.

2. World Health Organization. World Report on Vision. Geneva: World Health Organization, 2019.

3. Levtzion-Korach O, Tennenbaum A, Schnitzer R, Ornoy A. Early motor development of blind children. J Paediatr Child Health. 2000;36(3):226-9. doi: 10.1046/j.1440-1754.2000.00501.x. PubMed PMID: 10849221.

4. Chanfreau J, Andreas Cebulla A. Educational attainment of blind and partially sighted pupils. London: 2009.

5. Harrabi H, Aubin MJ, Zunzunegui MV, Haddad S, Freeman EE. Visual difficulty and employment status in the world. PLoS One. 2014;9(2):e88306. Epub 2014/02/07. doi: 10.1371/journal.pone.0088306. PubMed PMID: 24516632; PubMed Central PMCID: PMCPMC3917855.

6. Lamoureux EL, Hassell JB, Keeffe JE. The determinants of participation in activities of daily living in people with impaired vision. Am J Ophthalmol. 2004;137(2):265-70. Epub 2004/02/14. doi:

10.1016/j.ajo.2003.08.003. PubMed PMID: 14962415.

7. Legood R, Scuffham P, Cryer C. Are we blind to injuries in the visually impaired? A review of the literature. Injury Prevention. 2002;8(2):155-60. doi: 10.1136/ip.8.2.155. PubMed PMID: PMC1730864.

8. Lamoureux E, Gadgil S, Pesudovs K, Keeffe J, Fenwick E, Dirani M, et al. The relationship between visual function, duration and main causes of vision loss and falls in older people with low vision. Graefes Arch Clin Exp Ophthalmol. 2010;248(4):527-33. Epub 2010/01/08. doi: 10.1007/s00417-009-1260-x. PubMed PMID: 20054556.

9. Lord SR, Smith ST, Menant JC. Vision and falls in older people: risk factors and intervention strategies. Clin Geriatr Med. 2010;26(4):569-81. Epub 2010/10/12. doi: 10.1016/j.cger.2010.06.002. PubMed PMID: 20934611.

10. Ribeiro MV, Hasten-Reiter Junior HN, Ribeiro EA, Juca MJ, Barbosa FT, Sousa-Rodrigues CF. Association between visual impairment and depression in the elderly: a systematic review. Arq Bras Oftalmol. 2015;78(3):197-201. Epub 2015/07/30. doi: 10.5935/0004-2749.20150051. PubMed PMID: 26222114.

11. Jacobs JM, Hammerman-Rozenberg R, Maaravi Y, Cohen A, Stessman J. The impact of visual impairment on health, function and mortality. Aging Clin Exp Res. 2005;17(4):281-6. Epub 2005/11/16. PubMed PMID: 16285193.

12. World Health Organisation. Universal eye health: a global action plan 2014-2019. Geneva: World Health Organisation,, 2013.

13. United Nations. Convention on the Rights of Person's with Disabilities. Geneva2008.

14. United Nations Division for Social Policy and Development Disability. Sustainable Development Goals (SDGs) and Disability, [cited 2018 02/03/2018]. Available from:

https://www.un.org/development/desa/disabilities/about-us/sustainable-development-goals-sdgs-anddisability.html.

15. Virgili G. Reading aids for adults with low vision. 2018. PubMed PMID: 29664159.

16. Virgili G, Rubin G. Orientation and mobility training for adults with low vision. Cochrane Database of Systematic Reviews. 2010(5):CD003925. doi: https://dx.doi.org/10.1002/14651858.CD003925.pub3. PubMed PMID: 20464725.

17. van Nispen RMA, Virgili G, Hoeben M, Langelaan M, Klevering J, Keunen JEE, et al. Low vision rehabilitation for better quality of life in visually impaired adults. Cochrane Database of Systematic Reviews. 2020(1). doi: 10.1002/14651858.CD006543.pub2. PubMed PMID: CD006543. 
18. Binns AM, Bunce C, Dickinson C, Harper R, Tudor-Edwards R, Woodhouse M, et al. How effective is low vision service provision? A systematic review. Survey of Ophthalmology. 2012;57(1):34-65. doi: https://dx.doi.org/10.1016/i.survophthal.2011.06.006. PubMed PMID: 22018676.

19. Chiang PP, O'Connor PM, Le Mesurier RT, Keeffe JE. A global survey of low vision service provision. Ophthalmic Epidemiol. 2011;18(3):109-21. Epub 2011/05/26. doi: 10.3109/09286586.2011.560745. PubMed PMID: 21609239.

20. Moher D, Liberati A, Tetzlaff J, Altman DG. Preferred reporting items for systematic reviews and meta-analyses: the PRISMA statement. BMJ. 2009;339. doi: 10.1136/bmj.b2535.

21. The World Bank. World Bank Country and Lending Groups 2017 [cited 2017 10/10/2017]. Available from: https://datahelpdesk.worldbank.org/knowledgebase/articles/906519.

22. World Health Organisation. Rehabilitation: key for health in the 21st century 2017 [cited 2018 26/02/2018]. Available from: http://www.who.int/disabilities/care/KeyForHealth21stCentury.pdf?ua=1],. 23. Higgins JPT GSe. Cochrane Handbook for Systematic Reviews of Interventions Version 5.1.0 [updated March 2011] 2011. Available from: Available from http://handbook.cochrane.org.

24. Christy B. A Randomized Trial of Methods of Low Vision Service Delivery 2012.

25. Christy B, Keeffe JE, Nirmalan PK, Rao GN. A randomized controlled trial assessing the effectiveness of strategies delivering low vision rehabilitation: design and baseline characteristics of study participants. Ophthalmic Epidemiol. 2010;17(4):203-10. Epub 2010/07/21. doi: 10.3109/09286586.2010.483752. PubMed PMID: 20642342.

26. Atasavun Uysal S, Düger T. Visual perception training on social skills and activity performance in lowvision children. Scandinavian Journal of Occupational Therapy. 2012;19(1):33-41. doi: 10.3109/11038128.2011.582512. PubMed PMID: 104613305. Language: English. Entry Date: 20120117. Revision Date: 20150820. Publication Type: Journal Article.

27. Calik BB, Kitis A, Cavlak U, Oguzhanoglu A. The impact of attention training on children with low vision: a randomized trial. Turkish Journal of Medical Sciences. 2012;42(Sup. 1):1186-93. PubMed PMID: 20123362895.

28. Çolak T, Bamaç B, Aydin M, Meriç B, Özbek A. Physical fitness levels of blind and visually impaired goalball team players. Isokinetics \& Exercise Science. 2004;12(4):247-52. PubMed PMID: 106607987. Language: English. Entry Date: 20050422. Revision Date: 20150819. Publication Type: Journal Article. 29. Do AT, Ilango K, Ramasamy D, Kalidasan S, Balakrishnan V, Chang RT. Effectiveness of low vision services in improving patient quality of life at Aravind Eye Hospital. Indian Journal of Ophthalmology. 2014;62(12):1125-31. doi: 10.4103/0301-4738.149130. PubMed PMID: 109690880. Language: English. Entry Date: 20150605. Revision Date: 20170104. Publication Type: journal article. Journal Subset: Asia.

30. Gothwal VK, Sumalini R, Bharani S. Assessing the effectiveness of low Vision Rehabilitation in children: An observational study. Investigative Ophthalmology and Visual Science. 2015;56(5):3355-60. doi: http://dx.doi.org/10.1167/iovs.14-15760. PubMed PMID: 605664411.

31. Khan SA, Das T, Kumar SM, Nutheti R. Low vision rehabilitation in patients with age-related macular degeneration at a tertiary eye care centre in southern India. Clinical and Experimental Ophthalmology. 2002;30(6):404-10. doi: 10.1046/j.1442-9071.2002.00569.x. PubMed PMID: WOS:000179284600005. 32. Runjic T, Nikolic B, Bilic-Prcic A. The Impact of Rehabilitation on Mastering the Independent Mobility Technique of the Blind Veterans. [Croatian]. Hrvatska Revija Za Rehabilitacijska Istrazivanja. 2003;39(1):6371. PubMed PMID: 38009544.

33. Gothwal VK, Thomas R, Crossland M, Bharani S, Sharma S, Unwin H, et al. Randomized Trial of Tablet Computers for Education and Learning in Children and Young People with Low Vision. Optom Vis Sci. 2018;95(9):873-82. Epub 2018/08/29. doi: 10.1097/opx.0000000000001270. PubMed PMID: 30153236; PubMed Central PMCID: PMCPMC6133228.

34. Vijayakumar V, John RK, Datta D, Thulasiraj RD, Nirmalan PK. Quality of life after community-based rehabilitation for blind persons in a rural population of South India. Indian Journal of Ophthalmology. 2004;52(4):331-5. PubMed PMID: 40116037.

35. Jalali M, Sadjad Moussavi M, Yazdi A, Fadardi J. Effectiveness of Rational Emotive Behavior Therapy on Psychological Well-Being of People with Late Blindness2014. 
36. MS E, K A. Emotional intelligence and goal setting - an investigation into interventions to increase motivation to work among visually impaired students in Nigeria. British Journal of Visual Impairment.

444 2007;25(3):249-53. doi: 10.1177/0264619607079805.

445 37. Mahjoob M, Heravian J, Validad MH, Momeni Moghadam H, Hosini T, Tavakoli R. The effect of color 446 filters on the visual acuity and contrast sensitivity in low vision patients. [Arabic]. Journal of Babol University of Medical Sciences. 2010;11(6):53-7. PubMed PMID: 360040559.

38. Gothwal VK, Bharani S. Outcomes of Multidisciplinary Low Vision Rehabilitation in Adults. Investigative Ophthalmology \& Visual Science. 2015;56(12):7451-61. doi: https://dx.doi.org/10.1167/iovs.1516892. PubMed PMID: 26595605.

39. Aki E AS, Turan A, Kayihan H,. Training Motor Skills of Children with Low Vision. Perceptual and Motor Skills. 2007;104(3_suppl):1328-36. doi: 10.2466/pms.104.4.1328-1336.

40. Onuigbo LN, Eseadi C, Ebifa S, Ugwu UC, Onyishi CN, Oyeoku EK. Effect of Rational Emotive Behavior Therapy Program on Depressive Symptoms Among University Students with Blindness in Nigeria. Journal of Rational-Emotive \& Cognitive-Behavior Therapy. 2019;37(1):17-38. doi: 10.1007/s10942-018-0297-3.

41. Reeves B, Harper R, Russell W. Enhanced low vision rehabilitation for people with age related macular degeneration: A randomised controlled trial2004. 1443-9 p.

42. Chiang PP-C, Xie J, Keeffe JE. Identifying the Critical Success Factors in the Coverage of Low Vision Services Using the Classification Analysis and Regression Tree Methodology. Investigative Ophthalmology \& Visual Science. 2011;52(5):2790-5. doi: 10.1167/iovs.10-5460.

43. Bright T, Wallace S, Kuper H. A Systematic Review of Access to Rehabilitation for People with Disabilities in Low- and Middle-Income Countries. International journal of environmental research and public health. 2018;15(10):2165. doi: 10.3390/ijerph15102165. PubMed PMID: 30279358.

44. Crossland MD, Thomas R, Unwin H, Bharani S, Gothwal VK, Quartilho A, et al. Tablet computers versus optical aids to support education and learning in children and young people with low vision: protocol for a pilot randomised controlled trial, CREATE (Children Reading with Electronic Assistance To Educate). Bmj Open. 2017;7(6). doi: 10.1136/bmjopen-2017-015939. PubMed PMID: WOS:000406391200196.

45. World Health Organization. Rehabilitation 2030: a call for action. Geneva: World Health Organization, 2017. 
Table 1: PICO criteria

Population - Residents of LMICs

- Individuals whose best corrected visual acuity fulfils the ICD-10 criteria for moderate visual impairment, severe visual impairment or blindness (ICD-10 categories 1-5) in their best eye i.e. less than $6 / 18$ on a Snellen chart visual acuity (or equivalent).

- Adults or children

Intervention Eligible study designs:

- Randomised controlled trials

- Non-randomised controlled trials (controlled before-and-after trials or controlled interrupted time series)

Interventions 'that address impairments, activity limitations and participation restrictions, as well as personal and environmental factors (including assistive technology) that have an impact on functioning.' $(22,45)$ Excludes interventions which are able to fully correct visual impairment.

\section{Comparison - No intervention \\ - Best current practice \\ - Current service offer \\ - Other intervention}

Outcomes

- Visual Acuity

- Functional ability to carry out activities of daily living (e.g. mobilising, reading, writing etc.)

- Safety (accidents/incidents)

- Quality of life

- Psychological Outcomes*

*Addition from original protocol 
Table 2: Table of identified interventions in low- and middle-income countries

\section{DESIGN, PARTICIPANTS AND \\ SETTING}

\section{Aki et al. 2007 (39)}

Controlled before and after intervention study

$\mathrm{N}=40$ (20 in each group),

Children recruited from schoo

for the blind. They had severe

low vision (ICD-10). Mean age

training group 8 years 9

months, home training group 8

years 10 months. $50 \%$ male.

Ankara, Turkey

\section{Atasavun et al. 2012 (26)}

Randomised controlled tria

$\mathrm{N}=40$ (20 in each group)

children with low vision

(according to ICD-10) recruited

from a school for the blind. Age

7-14 years. $57.5 \%$ male

Ankara, Turkey AIM AND INTERVENTION

"To assess the effectiveness of a motor training program for visually impaired children"

Intervention: The training programme included coordination, balance, strength, visuomotor control and finger dexterity.

Training group: Administered by a

physiotherapist for 3 days/week for 1 hour per session, over 3 months.

Home training Group: parents taught the same programme.

Follow-up: Programme lasted for 3 months.

No follow-up after that described.

"To investigate and compare the effects of two Outcome measures: Motor free visual perception, social skills and activity performance.

different visual perception treatments on the

social skills and activity performance of low-

vision children"

Interventions: A visual perception training programme delivered two days/week for 45 minutes per session over 3 months.

Group 1: aided with paper and pen Group 2: aided with computer

Follow-up: Programme lasted for 3 months. No follow-up after that described.

\section{Calik et al. 2012 (27)}

Randomised Controlled Trial

$\mathrm{N}=20$ (10 in each group)

children recruited with low

vision (VA between 40/200 and

$2 / 200$ ) school for the visually

impaired. Age 7-12 years (mean

age 9.3 years group 1 and 10.4

years group 2). Sex not

reported

Denizili, Turkey
"To show the effectiveness of a 6 -week

attention training program on the cognition, quality of life (QOL), and activities of daily

living in children with low vision."

Intervention: An educational program (the Pay Attention $\odot$ training program) on 3 days/week (30 minutes per session) for 6 weeks.

Control group: no intervention.

Follow-up: Outcome was assessed at the end of the educational period ( 6 weeks). No follow up after that described.

\section{RESULTS}

\section{ASSESSMENT OF BIAS}

Outcome Measure: Motor skills (Bruininks-Oseretsky motor skills scale)

Before/after comparison: both groups scored better in 4 out of 5 of the Bruininks-Oseretsky motor skills scale assessment scores after training ( $p<0.05$ significance level), (not in visual motor control)

Between group comparison: No pre-test group comparisons done. Significant differences ( $p<0.05$ level) in favour of the training group (rather than home training group) at posttraining assessment in 5 of 8 domains of the motor skills assessment.

Random Sequence

Generation

Allocation

Concealment

Blinding of

Outcome

Assessment

Incomplete

Outcome Data

Selective Reporting

Selective Reporting

Random Sequence

Generation

Allocation

Before/after comparison:

- Motor-Free Visual Perception Test: Total visual perception scores significantly increased in both groups $(p<0.001)$, but not in all domains.

- Social Skills Assessment Tool for Children with Visual Impairment: Significant improvements overall and in all domains.

- Activity Performance Analysis (Canadian Occupational Performance Measure): Significant improvements reported in performance and total activities $(p<0.01)$, but not in satisfaction.

Between group comparison: No significant difference between the post-intervention visual perception of the two groups. $(p=0.18)$. Other outcomes between group comparison not reported.

Outcome measures: Modified child mini-mental state examination, activities of daily living questionnaire (NPI), vision-related quality of life (LV QOL).

Before/after measurement: Significant improvements were observed in the training group $(p<0.05)$ in all 3 domains, although not in every subtest. No significant changes were noted for the control group.

Between group comparison: There was significant improvements in the intervention group for all three domain totals, though not for all subsets (mean score intervention vs comparison):

- Modified child MMSE for cognitive function, 35.7 vs 30.6 ( $p=0.05$ )

Concealment

Blinding of

Outcome

Assessment

Incomplete

Outcome Data

Selective Reporting

2

\begin{tabular}{lc}
$\begin{array}{l}\text { Random Sequence } \\
\text { Generation }\end{array}$ & $\mathbf{2}$ \\
\hline $\begin{array}{l}\text { Allocation } \\
\text { Concealment }\end{array}$ & $\mathbf{2}$ \\
\hline $\begin{array}{l}\text { Blinding of } \\
\text { Outcome } \\
\text { Assessment }\end{array}$ & $\mathbf{3}$ \\
\hline $\begin{array}{l}\text { Incomplete } \\
\text { Outcome Data }\end{array}$ & $\mathbf{1}$ \\
\hline Selective Reporting & $\mathbf{1}$
\end{tabular}

Activities of Daily Living using the Northwick Park Index of Independence (NPI) score, 32.0 vs $29.8(p=0.04)$

Low Vision Quality of life (LVOOL) score 101.2 vs $84.4(p=0.03)$.

Risk of Bias: 1=low, 2=moderate, 3=high, ?=not described, VA=Visual Acuity 


\section{DESIGN, PARTICIPANTS AND \\ SETTING}

\section{Christy. 2012 (24)}

Four arm randomised

intervention trial.

$\mathrm{N}=436$ (109 in each arm, 393 completed study $(90 \%))$ permanent residents of two specified districts, recruited from first time referral patients at a tertiary care facility. Best corrected visual acuity $<6 / 12$ to light perception, or a visual field less than 20 degrees from the point of fixation in the better eye. $68.7 \%$ male. Adults and children (8-88 years).

Hyderabad, India

\section{Çolak et al. 2004 (28)}

Cross-sectional study

$\mathrm{N}=103$ (51 in intervention group and 52 in control group). VA $\leq 20 / 400$ or a visual field of $\leq 20$ degrees. Recruited from a school for the blind. $100 \%$ male. Average ages in the groups 14.3 to 15.8 years.

Istanbul, Turkey

\section{"To compare the effectiveness of low vision}

rehabilitation interventions delivered in four different arms"

Intervention: Included a range of low vision rehabilitation interventions (vocational rehabilitation, orientation and mobility, environment modifications, educational rehabilitation, use of low vision devices, computer assistive software, welfare services) which would all be delivered in one of the following four arms:

a) Centre-based rehabilitation

b) Community-based rehabilitation

c) Centre-based and community-based rehabilitation

d) Centre-based with non-interventional community visits

All initially included 3 consecutive days training.

Follow-up: Outcomes were assessed 9 months after the initial visit.

"To compare motor fitness levels between goalball players and non-goalball players with varying degrees of blindness."

Intervention: Playing goalball (a court-based team game with audible ball movements) for 6 hours per week. Duration unspecified.

Control: Non-active subjects not participating in any type of game before.

Follow-up: One off measurement.

\section{Outcomes measures:}

- Effectiveness of Low Vision Rehabilitation Training (ELVRT)

- Quality of life (WHOQOL)

- Adaptation to Vision Loss (AVL)

- Impact of visual Impairment (IVI) for Adults

- Impact of visual impairment (IVI) for Children

Before/after comparison: Overall there was a positive significant change for all outcome measures when participants from the four-arms were combined $(p<0.001)$. All four intervention groups show positive change for all outcome measures, although not all were statistically significant.

Between group comparison: The arms including a community element tended to demonstrate a larger effect size, but statistical comparisons were not presented.

\begin{tabular}{lc} 
Random & \\
$\begin{array}{l}\text { Sequence } \\
\text { Generation }\end{array}$ & $\mathbf{1}$ \\
\hline $\begin{array}{l}\text { Allocation } \\
\text { Concealment }\end{array}$ & $\mathbf{1}$ \\
\hline $\begin{array}{l}\text { Blinding of } \\
\text { Outcome } \\
\text { Assessment }\end{array}$ & $\mathbf{2}$ \\
\hline $\begin{array}{l}\text { Incomplete } \\
\text { Outcome Data }\end{array}$ & $\mathbf{2}$ \\
\hline $\begin{array}{l}\text { Selective } \\
\text { Reporting }\end{array}$ & $\mathbf{1}$ \\
\hline
\end{tabular}

\begin{tabular}{|c|c|c|}
\hline \multicolumn{3}{|c|}{$\begin{array}{l}\text { Sequence } \\
\text { Generation }\end{array}$} \\
\hline visual field of $\leq 5$ degrees], B3 [VA20/200-20/400 or a visual field or 5-20 degrees]) & $\begin{array}{l}\text { Allocation } \\
\text { Concealment }\end{array}$ & 3 \\
\hline $\begin{array}{l}\text { Before/after comparison: N/A } \\
\text { Between group comparison (intervention vs control): } \\
\text { - Significantly greater range of motion of shoulder, elbow and wrist }(p<0.05) \text { in the }\end{array}$ & $\begin{array}{l}\text { Blinding of } \\
\text { Outcome } \\
\text { Assessment }\end{array}$ & 2 \\
\hline $\begin{array}{l}\text { intervention group found in most comparisons. } \\
\text { - Balance response: Significantly greater balance duration ( } p=0.01 \text { or less) in all three }\end{array}$ & $\begin{array}{l}\text { Incomplete } \\
\text { Outcome Data }\end{array}$ & n/a \\
\hline $\begin{array}{l}\text { groups } \\
\text { - Torque strength of shoulder rotation: significantly greater for internal rotation for all }\end{array}$ & $\begin{array}{l}\text { Selective } \\
\text { Reporting }\end{array}$ & 1 \\
\hline
\end{tabular}

- Torque strength of shoulder rotation: significantly great

- Vertical jump: Significantly greater in the intervention group.

- Handgrip strength: Significant differences in B2 and B3, but not B1 group

- Sit and reach: Significantly greater reach distance for B1 and B2 but not for B3.

Risk of Bias: 1=low, 2=moderate, 3=high, ?=not described, n/a=not applicable, VA=Visual Acuity 


\section{DESIGN, PARTICIPANTS AND}

SETTING

\section{Do et al. 2014 (29)}

Controlled before and after

intervention study

$\mathrm{N}=44$ completed the study $(24$

received low vision aids). VA $<20 / 70$

to light perception in the better eye

or visual fields $<10^{\circ}$ from the point of

fixation. 57\% male. Age between 10 and 70 years old.

\section{Madurai, India}

\section{Eniola et al. 2007 (36)}

Two armed before and after intervention study

$\mathrm{N}=32$ (16 in each group), children who were randomly selected from a School for "Handicapped" Children. VA criteria not given. $44 \%$ male, age not reported.

Ibadan and Osogbo, Nigeria

\section{Gothwal at al. 2015 (30)}

Controlled before and after intervention study.

$\mathrm{N}=183$ completed the programme (397 recruited). Included children with VA of $<20 / 60$ or visual field of $<20$ degrees in the better eye. $57 \%$ male. Mean age 11.9 years.

Hyderabad, India
"To survey the effectiveness of low vision exams and visual aids in improving patient quality of life in southern rural India"

Intervention: Provision of low vision aids (including hand or stand magnifiers, spectacle magnifiers, telescopes, closed-circuit televisions, and tinted spectacles). Low vision aids were provided to patients depending on whether their vision improved with any aids, or they refused, and depended on disease type acuity level and mental capacity.

Control: Low vision aid not provided.

Follow-up: 1 month after first visit

\section{"To explore the impact of emotional intelligence and goal setting} techniques upon the motivation to work among visually impaired students."

Interventions: Either

- Goal setting intervention, or

- Emotional Intelligence intervention

Lectures, discussions, demonstrations and take-home activities were all used. The study was carried out over 6 weeks, with 2 sessions per week.

Follow-up: Outcomes measured at the end of the 6 week course. No follow-up after that described.

"To evaluate the change in visual functioning (VF) using the L. V. Prasad-Functional Vision Questionnaire II (LVP-FVQ II) following multidisciplinary low vision rehabilitation (LVR) services in children with low vision"

Intervention: Multidisciplinary low vision rehabilitation service.

Control: Children non-compliant with intervention.

Follow-up: 3-4 months from baseline appointment
Outcome measure: Vision-related quality of Life (LV QOL).

Before/After measurement: Among the low vision aids group

VOOL improved 8.89 points $(p<0.001)$ while in the control group reduced by -0.65 points $(p=0.32)$

Between group comparison: Not reported post-intervention. No significant difference between the groups pre-intervention.

\section{Outcome measure: Motivation (Work value inventory (WVI)} questionnaire)

Before/after comparison: A significant improvement in the mean level of motivation was found in both arms $(p<0.05)$ : Emotional intelligence 7.7 to 17.9 and Goal Setting 11.1 to 14.0 .

Between group comparison: No significant difference was found between the two groups.
Generation

Allocation

Concealment

Blinding of Outcome

Assessment

Incomplete

Outcome Data

Selective Reporting 1

Risk of Bias: 1=low, 2=moderate, 3=high, ?=not described, n/a=not applicable, VA=Visual Acuity

\section{Outcome measure: Visual Functioning (LVP-FVQ II).}

Before/after comparison:

- Overall: the post-rehabilitation score was significantly improved compared with the pre-rehabilitation score $(-2.53$ vs -1.33 $p<0.0001)$.

- Compliant group: Significant improvement (-1.22 to -

$3.44, p<0.0001$

- Non-compliant group: No change $(-1.46$ to $-1.41, p<0.71)$

Between group comparison: Visual functioning is significantly

better in the compliant group compared with non-compliant group $(p<0.0001)$

\begin{tabular}{lc}
$\begin{array}{l}\text { Random Sequence } \\
\text { Generation }\end{array}$ & $?$ \\
\hline $\begin{array}{l}\text { Allocation } \\
\text { Concealment }\end{array}$ & $?$ \\
\hline $\begin{array}{l}\text { Blinding of Outcome } \\
\text { Assessment }\end{array}$ & $?$ \\
\hline $\begin{array}{l}\text { Incomplete } \\
\text { Outcome Data }\end{array}$ & $\mathbf{1}$ \\
\hline Selective Reporting & $\mathbf{1}$ \\
\hline
\end{tabular}

\begin{tabular}{lc}
$\begin{array}{l}\text { Random Sequence } \\
\text { Generation }\end{array}$ & $\mathbf{3}$ \\
\hline $\begin{array}{l}\text { Allocation } \\
\text { Concealment }\end{array}$ & $\mathbf{3}$ \\
\hline $\begin{array}{l}\text { Blinding of Outcome } \\
\text { Assessment }\end{array}$ & $\mathbf{2}$ \\
\hline $\begin{array}{l}\text { Incomplete } \\
\text { Outcome Data }\end{array}$ & $\mathbf{3}$ \\
\hline Selective Reporting & $\mathbf{1}$ \\
\hline
\end{tabular}




\section{DESIGN, PARTICIPANTS AND \\ SETTING}

Gothwal at al. 2015 (38)

Two-armed before and after

intervention study

$\mathrm{N}=255$ completed the programme

(1271 recruited). Included adults with

VA of $\geq 20 / 60$ to $<20 / 200$ or visual

field of $<20$ degrees in the better eye.

$77 \%$ male, mean age 41.7 years.

Hyderabad, India

\section{Gothwal et al. 2019 (33)*}

Randomised controlled trial. (pilot study)

$\mathrm{N}=20 *$ Best corrected VA of $\angle 6 / 18$ to $3 / 60$ in the better eye. $55 \%$ male. Children aged $10-18$ years (mean:

14.2 years control, 13.4 years intervention).

Hyderabad, India
"To evaluate the outcomes of multidisciplinary low vision rehabilitation (LVR) in adults with low vision in India using the Veterans Affairs Low Vision Visual Functioning Questionnaire (VA LV VFQ-48) and the Impact of Vision Impairment (IVI) questionnaire"

Intervention: Multidisciplinary low vision

rehabilitation service with subgroup comparison of

optometry led vs other services.

Follow-up: 4 months from baseline appointment

The study had the "primary objective of

determining whether a full randomized controlled

trial of tablet computers as assistive technology to

support education would be feasible. Secondary

objectives were to explore acceptability,

accessibility, and any changes in vision-related

quality of life, functional vision, and measures of

reading speed, accuracy, and comprehension."

Intervention: Tablet computers with low-vision applications.

Control: Conventional low-vision support as per standard clinical care, which includes optical LVA and/or CCTV.

Follow-up: 3 months and 6 months after baseline
RESULTS

ASSESSMENT OF BIAS

Outcome measures:

- Vision-related quality of life (Impact of vision Impairment, IVI)

Visual functioning (Veterans Affair Low Vision Visual Functioning Questionnaire-48, VA LV VFQ-48)

Before/After comparison:

- IVI: Significant improvement $(\mathrm{P}<0.0001)$ overall and for the subscales of mobility and independence, and reading and accessing information, but not significant in the emotional wellbeing subscale $(p=0.06)$

- VA LV VFQ-48: Significant improvements $(p<0.0001)$ overall and in all subscales.

Between group comparison: No difference in impact by type of service (optometry led or other services), (data not provided)

Outcome measures:

- Functional visual ability (LV Prasad Functional Vision Questionnaire (LVP-FVQ II).

- Vision-related quality of life (Impact of Vision Impairment for Children

Questionnaire).

- Critical print size

Before/after comparison (India only): No significant change from baseline to 3 or 6 months in any outcome measure.

Between group comparison: Not reported
Random Sequence

Allocation

Concealment

Blinding of

Outcome

Assessment

Incomplete

Outcome Data

Selective Reporting

Random Sequence 1

Generation

Allocation

Concealment

Blinding of

Outcome

Assessment

Incomplete

Outcome Data

Selective Reportin

Other: Study partly funded

by device manufacturer

Risk of Bias: 1=low, 2=moderate, 3=high, ?=not described, VA=Visual Acuity

*There were also 20 children recruited from UK (London and Bedford), outcomes from India arm only reported 


\section{DESIGN, PARTICIPANTS AND \\ SETTING}

\section{Jalali et al. 2014 (35)}

Randomised controlled trial

$\mathrm{N}=60$ (30 in each group)

Adults who were 'late blind and

partially sighted' recruited from

rehabilitation and training centres for

the blind and from NGOs. VA criteria

not reported. Both males and females

included but sex distribution not

reported. Age 20-40 years.

Mashhad, Iran

\section{Khan et al. 2002 (31)}

Controlled before and after

intervention study

$\mathrm{N}=100$, Adults ( $\geq 45$ years) with agerelated macular degeneration. For distance vision, 36 patients received spectacles and 7 patients received telescopes, including one who received both. VA criteria not given. $73 \%$ male and mean age 69.2 years.

Hyderabad, India

\section{Onuigbo et al. 2018 (40)}

Randomised controlled trial

$\mathrm{N}=65$ University students with blindness who scored at least 20 on

the Beck Depression Inventory-II. 46\%

Male. Mean age 25.6 years

intervention, 25.3 years control.

South East Zone, Nigeria
"To investigate the effectiveness of Rational Emotive Behaviour Therapy (REBT) on improving the psychological wellbeing of people with late blindness."

Intervention: Intervention group received therapist-led training in rational emotive beliefs therapy (REBT). No detail of dose or timing. Control: No information provided on the control group (although noted that no one received placebo treatment

Follow-up: Post-course and 1 month after the end of the course.

"The aim of this study was to evaluate the specific needs and types of low vision devices (LVDs) in patients with AMD [age-related macular degeneration]."

Intervention: Rehabilitation service including education on AMD, eccentric viewing techniques, provision of low vision devices (LVD) and psychosocial counselling.

Control: As above, but with prescription of standard spectacles rather than LVD.

Follow-up: Not defined

To "examine the efficacy of group-based rational emotive behaviour therapy (REBT) intervention on depressive symptoms among selected university students with blindness in Nigeria"

Intervention: 12 week REBT course, with 2 weekly follow-up for 2 months.

Control: No intervention.

Follow-up: Post-course and after 2 months follow-
Outcome measures: Psychological wellbeing (Jones Irrational Beliefs Questionnaire, Inventory of Depression, Anxiety and Stress (DASS21), Eysenck's Self Esteem Inventory).

Before/After comparison: 341 to post-test 234) and in all subscales of the irrational beliefs questionnaire $(p<0.001)$, but no difference observed in the control group ( $p>0.1)$. The findings appear to be sustained from post-test to 1 month follow-up. Significant reduction in the intervention group in measures of depression, anxiety and stress $(p<0.001)$, and improvement in self-esteem $(p<0.001)$ but no difference observed in the control group $(p>0.4)$. The findings appear to be sustained from post-test to 1 month follow-up.

Outcome: Visual Acuity.

Before/after comparison:

- With standard spectacles, patients with visual acuity $<6 / 18$ reduced from $72.2 \%(26 / 36)$ to $47.2 \%(17 / 36)(p=0.03)$

- With a telescope, visual acuity $<6 / 18$ reduced from $85.7 \%(6 / 7)$ to $14.3 \%(1 / 7)$ $(p=0.029)$.

Between group comparison: Not reported
Significant improvement in the intervention group overall (mean score pre-test

\section{Random Sequence}

Generation

Allocation

Concealment

Blinding of

Outcome

Assessment

Incomplete

Outcome Data

Outcome Data
Selective Reporting $\mathbf{1}$

Random Sequence $\mathbf{3}$

Allocation

Concealment

Blinding of

Outcome

Assessment

Incomplete

Outcome Data

Selective Reporting 1

\section{Main outcome measure: Beck Depression Inventory-II score}

Before/after comparison: Significant reduction in depression score pre-post course (30.8 to $12.9 \mathrm{p}<0.001$ ) in the intervention group. No change seen in control group ( 32.3 to $32.3, p=0.87$ ). Between post-course and at 2 month follow-up, there was a significant reduction in score in the intervention group (12.9 to $10.0, p=0.002$ ), but not in the control group (32.3 to $31.8, p=0.81$ ).

Between group comparison: There no significant difference between the baseline scores ( 30.8 and $32.3, p=0.72$ ), but there was a significant difference between the two groups post-test $(12.9$ and $32.3, p<0.001)$ and at the end of follow-up (31.8 and 10.0, $p<0.001)$.

\begin{tabular}{lc}
$\begin{array}{l}\text { Random Sequence } \\
\text { Generation }\end{array}$ & $\mathbf{1}$ \\
\hline $\begin{array}{l}\text { Allocation } \\
\text { Concealment }\end{array}$ & $\mathbf{2}$ \\
\hline $\begin{array}{l}\text { Blinding of } \\
\text { Outcome } \\
\text { Assessment }\end{array}$ & $?$ \\
\hline $\begin{array}{l}\text { Incomplete } \\
\text { Outcome Data }\end{array}$ & $\mathbf{1}$ \\
\hline Selective Reporting & $\mathbf{1}$ \\
\hline $\begin{array}{l}\text { Other: Intervention group } \\
\text { only given travel expenses }\end{array}$
\end{tabular}

Risk of Bias: 1=low, 2=moderate, 3=high, ?=not described, VA=Visual Acuity 


\section{DESIGN, PARTICIPANTS AND \\ SETTING}

Runjic et al. 2003 (32)

Non-randomised controlled trial

$\mathrm{N}=28$ * Blind or partially sighted war veterans recruited at

rehabilitation centre. 11

individuals were diagnosed with

PTSD. VA criteria not reported.

Age and sex not reported.

Zagreb, Croatia

Vijayakumar et al. 2004 (34)

Controlled before and after

intervention study

$\mathrm{N}=159$ (84 received economic rehabilitation) blind individuals defined as best corrected

VA<3/60. 46.5\% male. Age $\geq 15$ years, mean age of participants 45.0 years.

"To determine to what extent the process of rehabilitation contributed to mastering of the essential independent mobility techniques."

Intervention: a rehabilitation course delivered at a centre. No further description.

Control: did not receive the rehabilitation course.

Follow-up: Not Reported

* States 20 completed the course - unclear whether the remaining 8 are participants who dropped out or the comparison group

"To determine the impact of community-based rehabilitation on the quality of life on a rural South Indian Population.

Intervention: Community-based rehabilitation and economic rehabilitation "focussed on providing skills to run a trade or pursue a profession" e.g. agriculture training, setting up small business ventures, animal rearing and crafts. Included some "monetary compensation".

Control: Community Based Rehabilitation only

Follow-up: 6 months after rehabilitation

Theni District, India
Main outcome measure: Independent mobility (tool not described)

Before/after comparison: Not reported.

Between group comparison: Independent mobility was significantly better in the intervention group compared to the control group $(p<0.001)$ after the intervention.

Main outcome measure: Quality of life (12 item quality of
life instrument).

Before/after comparison: Overall improvement in quality of life for $95.0 \%$ of individuals, worsened in $4.0 \%$ and remained life for $95.0 \%$ of individuals, worsened in $4.0 \%$ and remain (difference in mean scores divided by standard deviation at baseline) was 2.36 (95\% Cl approx. 2.05 to 2.65) for overall quality of life, with anything greater than 0.8 regarded as a large effect. Not reported separately for those with/without economic rehabilitation.

Between group comparison: There was no significant difference in quality of life between those who did and did not receive economic rehabilitation $\mathrm{p}=0.1$. Details not given
Random Sequence

eneration

Allocation

Concealment

Blinding of

Outcome

Assessment

Incomplete

Outcome Data

Selective Reporting ?

Random Sequence

Generation

Allocation

Concealment

Blinding of

Outcome

3

Assessment

Outcome Data

Selective Reporting

Risk of Bias: 1=low, 2=moderate, 3=high, ?=not described, VA=Visual Acuity 
